\title{
On a Couple of Nonlocal Singular Viscoelastic Equations with Damping and General Source Terms: Blow-Up of Solutions
}

\author{
Erhan Piskin, ${ }^{1}$ Salah Mahmoud Boulaaras (D, ${ }^{2,3}$ Hasan Kandemir, ${ }^{1}$ \\ Bahri Belkacem Cherif $\mathbb{D}^{2,4}$ and Mohamed Biomy ${ }^{2,5}$ \\ ${ }^{1}$ Dicle University, Faculty of Education Mathematics, Diyarbakir, Turkey \\ ${ }^{2}$ Department of Mathematics, College of Sciences and Arts, Ar Rass, Qassim University, Saudi Arabia \\ ${ }^{3}$ Laboratory of Fundamental and Applied Mathematics of Oran (LMFAO), University of Oran 1, Oran, 31000 Oran, Algeria \\ ${ }^{4}$ Preparatory Institute for Engineering Studies in Sfax, Tunisia \\ ${ }^{5}$ Department of Mathematics and Computer Science, Faculty of Science, Port Said University, Port Said 42511, Egypt
}

Correspondence should be addressed to Salah Mahmoud Boulaaras; s.boularas@qu.edu.sa

Received 28 March 2021; Revised 13 July 2021; Accepted 8 August 2021; Published 23 August 2021

Academic Editor: Santosh Kumar

Copyright (C) 2021 Erhan Piskin et al. This is an open access article distributed under the Creative Commons Attribution License, which permits unrestricted use, distribution, and reproduction in any medium, provided the original work is properly cited.

Under some given conditions, we prove the explosion result of the solution of the system of nonlocal singular viscoelastic with damping and source terms on general case. This current study is a general case of the previous work of Boulaaras.

\section{Introduction}

During the last decades, many nonlocal problems of deterministic and parabolic partial differential equations have been studied. These equations and their systems represent the modeling of many physical phenomena related to time. These constraints can be data measured directly at the boundary or give integral boundary conditions (for instance, see $[1-25])$.

In this work, we investigate the blow-up of the following system of nonlinear damping term:

$$
\left\{\begin{array}{l}
u_{t t}-\frac{1}{x}\left(x u_{x}\right)_{x}+\int_{0}^{t} g_{1}(t-s) \frac{1}{x}\left(x u_{x}(x, s)\right)_{x} \mathrm{~d} s+\left|u_{t}\right|^{m-1} u_{t}=f_{1}(u, v), Q, \\
v_{t t}-\frac{1}{x}\left(x v_{x}\right)_{x}+\int_{0}^{t} g_{2}(t-s) \frac{1}{x}\left(x v_{x}(x, s)\right)_{x} \mathrm{~d} s+\left|v_{t}\right|^{m-1} v_{t}=f_{2}(u, v), Q \\
u(x, 0)=u_{0}(x), u_{t}(x, 0)=u_{1}(x), \quad x \in(0, \alpha), \\
v(x, 0)=v_{0}(x), v_{t}(x, 0)=v_{1}(x), \quad x \in(0, \alpha), \\
u(\alpha, t)=v(\alpha, t)=0, \int_{0}^{\alpha} x u(x, t) \mathrm{d} x=\int_{0}^{\alpha} x v(x, t) \mathrm{d} x=0,
\end{array}\right.
$$

where $f_{1}(u, v), f_{2}(u, v): R^{2} \longrightarrow R$ given by

$$
\begin{aligned}
& f_{1}(u, v)=a|u+v|^{2(r+1)}(u+v)+b|u|^{r} u|v|^{r+2}, \\
& f_{2}(u, v)=a|u+v|^{2(r+1)}(u+v)+b|v|^{r} v|u|^{r+2},
\end{aligned}
$$

with $a, b \in R, r \geq-1$ (we get $a=b=1$ ), $Q=(0, \alpha) \times(0, T), \alpha$ $<\infty, T<\infty$, and

$$
g_{1}(.), g_{2}(.): R^{+} \longrightarrow R^{+}
$$

are given functions which will be specified later. The motivation of our work is because of some results regarding the following research paper: in [12], under some conditions suitable for the relaxation function, the author explained that solutions with initial negative energy explode in a finite time if $p>m$ and continue to find if $m \geq p$, for the following studied problem:

$$
\left\{\begin{array}{l}
u_{t t}-\Delta u+\int_{0}^{t} g(t-s) \Delta u+u_{t}\left|u_{t}\right|^{m-2}=|u|^{p-2} u, \quad \text { in } \Omega \times(0, \infty), \\
u=0 x \in \partial \Omega, \quad t \in(0, \infty), \\
u(x, 0)=u_{0}(x), u_{t}(x, 0)=u_{1}(x), \quad x \in \Omega .
\end{array}\right.
$$

In [4], the author studied a model describing the 
movement of a flexible two-dimensional viscous body on the unit disk (i.e., radial solutions) and by using some density arguments and some prior estimates, the authors demonstrated the existence and uniqueness of a generalized solution to the following problem:

$\left\{\begin{array}{l}u_{t t}-\frac{1}{x}\left(x u_{x}\right)_{x}+\int_{0}^{t} g(t-s) \frac{1}{x}\left(x u_{x}(x, s)\right)_{x} \mathrm{~d} s=f\left(x, t, u, u_{x}\right), \quad \text { in } Q, u_{x}(1, t)=0, \\ \int_{0}^{1} x u(x, t) \mathrm{d} x=0, \quad t \in(0, T), \\ u(x, 0)=\varphi(x), \\ u_{t}(x, 0)=\psi(x), \quad x \in(0,1),\end{array}\right.$

where

$$
Q=(0,1) \times(0, T)
$$

and $f$ is the right-hand side that satisfied the Lipschitzian condition. Recently, in [3], the authors demonstrated the decay result of energy for a small enough initial data together with the explosion result of large initial data of the following singular problem:

$$
\left\{\begin{array}{l}
u_{t t}-\frac{1}{x}\left(x u_{x}\right)_{x}+\int_{0}^{t} g(t-s) \frac{1}{x}\left(x u_{x}(x, s)\right)_{x} \mathrm{~d} s=|u|^{p-2} u \\
u(a, t)=0, \int_{0}^{a} x u(x, t) \mathrm{d} x=0 \\
u(x, 0)=\varphi(x), u_{t}(x, 0)=\psi(x) .
\end{array}\right.
$$

That is, they obtained the blow-up properties of local solution by Georgiev-Todorova method with nonpositive initial energy. More work followed up on similar nonlocal singular viscoelastic equations and systems in $[8,9]$.

In this work, we continue the study on system (1). According to some given conditions, we prove the explosion result of the solution of the system of nonlocal singular viscoelastic with damping and source terms on general case, where we begin by giving basic definitions and theories about the function spaces we need, and then, we mention the theorem of local existence. Finally, we announce and prove the main result of our studied problem in (1).

1.1. Preliminaries. In this section, we introduce some functional spaces and give some lemma's need for the remaining of this paper. Let $L_{x}^{p}=L_{x}^{p}((0, \alpha))$ be the weighed Banach space equipped with the norm

$$
\|u\|_{L_{x}^{p}}=\left(\int_{0}^{\alpha} x|u|^{p} \mathrm{~d} x\right)^{1 / p} .
$$

$H=L_{x}^{2}((0, \alpha))$ is, in particular, the Hilbert space of square integral functions having the finite norm

$$
\|u\|_{H}=\left(\int_{0}^{\alpha} x u^{2} \mathrm{~d} x\right)^{1 / 2}
$$

$V=V_{x}^{1}((0, \alpha))$ is the Hilbert space equipped with the norm

$$
\begin{gathered}
\|u\|_{V}=\left(\|u\|_{H}^{2}+\left\|u_{x}\right\|_{H}^{2}\right)^{1 / 2} \\
V_{0}=\{u \in V: u(\alpha)=0\} .
\end{gathered}
$$

Lemma 1 (Poincare-type inequality). For any $u \in V_{0}$,

$$
\int_{0}^{\alpha} x u^{2} d x \leq C_{P} \int_{0}^{\alpha} x u_{x}^{2} d x
$$

where $C_{P}$ is some positive constant.

Remark 2. It is clear that $\|u\|_{V_{0}}=\left\|u_{x}\right\|_{H}$ defines an equivalent norm on $V_{0}$.

Lemma 3. For any $u \in V_{0}$ and $2<p<4$, we have

$$
\|u\|_{L_{x}^{p}(0, \alpha)}^{p} \leq C_{*}\left\|u_{x}\right\|_{H=L_{x}^{2}(0, \alpha)}^{p}
$$

where $C_{*}$ is a constant depending on $\alpha$ and $p$ only. For the $g_{1}$ and $g_{2}$ functions, assumptions are as follows:(G1): $g_{1}(),. g_{2}($ .): $R^{+} \longrightarrow R^{+}$are two differentiable and nonincreasing functions with

$$
\begin{aligned}
& g_{1}(t) \geq 0,1-\int_{0}^{\infty} g_{1}(s) d s=I_{1} \geq 0, \\
& g_{2}(t) \geq 0,1-\int_{0}^{\infty} g_{2}(s) d s=I_{2} \geq 0,
\end{aligned}
$$

(G2): For all $t \geq 0$,

$$
\begin{aligned}
& g_{1}(t) \geq 0, \\
& g_{1}^{\prime}(t) \leq 0, \\
& g_{2}(t) \geq 0, \\
& g_{2}^{\prime}(t) \leq 0 .
\end{aligned}
$$

(G3): $r \geq-1$.

Theorem 4. Suppose that (G1), (G2), and (G3) hold. Then, for all $\left(u_{0}, v_{0}\right) \in V_{0}^{2}$ and all $\left(u_{1}, v_{1}\right) \in H^{2}$, problem $((1))$ admits a unique local solution $(u, v)$ :

$$
u, v \in C\left((0, T) ; V_{0}\right) \cap C^{l}((0, T) ; H),
$$

for $T>0$ small enough. 
Lemma 5. Assume that (G1), (G2), and (G3) hold and (u,v) is a solution of problem((1)); then, the energy functional

$$
\begin{aligned}
E(t)= & \frac{1}{2} \int_{0}^{\alpha} x u_{t}^{2} d x+\frac{1}{2} \int_{0}^{\alpha} x v_{t}^{2} d x+\frac{1}{2}\left(1-\int_{0}^{t} g_{1}(s) d s\right) \int_{0}^{\alpha} x u_{x}^{2} d x \\
& +\frac{1}{2}\left(1-\int_{0}^{t} g_{2}(s) d s\right) \int_{0}^{\alpha} x v_{x}^{2} d x+\frac{1}{2}\left(g_{1} \circ u_{x}\right)(t) \\
& +\frac{1}{2}\left(g_{2} \circ v_{x}\right)(t)-\int_{0}^{\alpha} x F(u, v) d x,
\end{aligned}
$$

where

$$
\begin{aligned}
\left(g_{1} \circ u_{x}\right)(t) & =\int_{0}^{\alpha} \int_{0}^{t} x g_{1}(t-s)\left|u_{x}(x, s)-u_{x}(x, t)\right|^{2} d s d x \\
\left(g_{2} \circ v_{x}\right)(t) & =\int_{0}^{\alpha} \int_{0}^{t} x g_{2}(t-s)\left|v_{x}(x, s)-v_{x}(x, t)\right|^{2} d s d x \\
F(u, v) & =\frac{1}{2(r+2)}\left[|u+v|^{2(r+2)}+2|u v|^{r+2}\right]
\end{aligned}
$$

Remark 6. Multiplying the first equation in ((1))by $x u_{t}$ and the second equation in((1))by $x v_{t}$ integrating over $(0, \alpha)$, we obtain the following equation:

$$
\begin{aligned}
\frac{d}{d t}[E(t)] & =-\int_{0}^{\alpha} x\left|u_{t}\right|^{m+1} \mathrm{~d} x-\int_{0}^{\alpha} x\left|v_{t}\right|^{m+1} \mathrm{~d} x, \\
& =-\left[\left\|u_{t}\right\|_{L_{x}^{m+1}}^{m+1}+\left\|v_{t}\right\|_{L_{x}^{m+1}}^{m+1}\right] .
\end{aligned}
$$

The definition of the norm is as follows:

$$
\left[\left\|u_{t}\right\|_{L_{x}^{m+1}}^{m+1}+\left\|v_{t}\right\|_{L_{x}^{m+1}}^{m+1}\right] \geq 0
$$

From here,

$$
-\left[\left\|u_{t}\right\|_{L_{x}^{m+1}}^{m+1}+\left\|v_{t}\right\|_{L_{x}^{m+1}}^{m+1}\right] \leq 0
$$

Thus,

$$
\frac{d}{d t}[E(t)]=-\left[\left\|u_{t}\right\|_{L_{x}^{m+1}}^{m+1}+\left\|v_{t}\right\|_{L_{x}^{m+1}}^{m+1}\right] \leq 0 .
$$

Lemma 7. There exist $c_{0}$ and $c_{1}$ positive constants such that $\frac{c_{0}}{2(r+2)}\left(|u|^{2(r+2)}+|v|^{2(r+2)}\right) \leq F(u, v) \leq \frac{c_{1}}{2(r+2)}\left(|u|^{2(r+2)}+|v|^{2(r+2)}\right)$.

Lemma 8. If $2 \leq s \leq p$,

$$
\|u\|_{L_{x}^{p}}^{s} \leq C\left(\left\|u_{x}\right\|_{H}^{2}+\|u\|_{L_{x}^{p}}^{p}\right)
$$

\section{Blow-Up of Solution}

In this section, we shall deal with the blow-up behavior of solutions for problem (1). We derive the blow-up properties of solutions of problem (1) with nonpositive initial energy by the method given in [1].

Theorem 9. Assume that (G1), (G2), and (G3) hold. $E(0)<0$ and

$$
\int_{0}^{\infty} g_{i}(s) d s<\frac{r+1}{r+1+1 /(4(r+2))}, i=1,2
$$

Then, the solution of problem (1) blows up in finite time.

Proof. Since $(d / d t)[E(t)]=E^{\prime}(t) \leq 0$,

$$
E(t) \leq E(0)<0, \quad \forall t \geq 0
$$

We define $H(t)=-E(t)$; then,

$$
0<H(0) \leq H(t)=-E(t), \quad \forall t \geq 0 .
$$

We obviously substitute $E(t)$ in (26); then,

$$
\begin{aligned}
0<H(0) \leq H(t)= & -\frac{1}{2} \int_{0}^{\alpha} x u_{t}^{2} \mathrm{~d} x-\frac{1}{2} \int_{0}^{\alpha} x v_{t}^{2} \mathrm{~d} x \\
& -\frac{1}{2}\left(1-\int_{0}^{t} g_{1}(s) \mathrm{d} s\right) \int_{0}^{\alpha} x u_{x}^{2} \mathrm{~d} x \\
& -\frac{1}{2}\left(1-\int_{0}^{t} g_{2}(s) \mathrm{d} s\right) \int_{0}^{\alpha} x v_{x}^{2} \mathrm{~d} x \\
& -\frac{1}{2}\left(g_{1} \circ u_{x}\right)(t)-\frac{1}{2}\left(g_{2} \circ v_{x}\right)(t) \\
& +\int_{0}^{\alpha} x F(u, v) \mathrm{d} x .
\end{aligned}
$$

From (22) and (27),

$$
\begin{aligned}
0<H(0) & \leq H(t) \leq \int_{0}^{\alpha} x F(u, v) \mathrm{d} x \\
& \leq \frac{c_{1}}{2(r+2)}\left[\int_{0}^{\alpha} x|u|^{2(r+2)} \mathrm{d} x+\int_{0}^{\alpha} x|v|^{2(r+2)} \mathrm{d} x\right] \\
& =\frac{c_{1}}{2(r+2)}\left[\|u\|_{L_{x}^{2(r+2)}}^{2(r+2)}+\|v\|_{L_{x}^{2(r+2)}}^{2(r+2)}\right] .
\end{aligned}
$$

Thus,

$$
H(t) \leq \frac{c_{1}}{2(r+2)}\left[\|u\|_{L_{x}^{2(r+2)}}^{2(r+2)}+\|v\|_{L_{x}^{2(r+2)}}^{2(r+2)}\right]
$$

Equation (29) will then be used as an important data for proof of the theorem. Now, we define 


$$
L(t)=H^{1-\sigma}(t)+\varepsilon\left(\int_{0}^{\alpha} x u u_{t} \mathrm{~d} x+\int_{0}^{\alpha} x v v_{t} \mathrm{~d} x\right)
$$

for $\varepsilon$ small enough and

$$
0<\sigma \leq \min \left\{\frac{2(r+2)-\mathrm{m}}{2 m(r+2)}, \frac{2(r+2)-m}{2 m(r+2)}, \frac{2 r+2}{4(r+2)}\right\} \text {. }
$$

By differentiating (30), using (1) and $H^{\prime}(t)=\left\|u_{t}\right\|_{L_{x}^{m+1}}^{m+1}$ $+\left\|v_{t}\right\|_{L_{x}^{m+1}}^{m+1}$, we obtain

$$
\begin{aligned}
L^{\prime}(t)= & (1-\sigma) H^{-\sigma}(t)\left[\left\|u_{t}\right\|_{L_{x}^{m+1}}^{m+1}+\left\|v_{t}\right\|_{L_{x}^{m+1}}^{m+1}\right]+\varepsilon \int_{0}^{\alpha} x u_{t}^{2} \mathrm{~d} x \\
& +\varepsilon \int_{0}^{\alpha} x v_{t}^{2} \mathrm{~d} x-\varepsilon\left(1-\int_{0}^{t} g_{1}(s) \mathrm{d} s\right) \int_{0}^{\alpha} x u_{x}^{2} \mathrm{~d} x \\
& -\varepsilon\left(1-\int_{0}^{t} g_{2}(s) \mathrm{d} s\right) \int_{0}^{\alpha} x v_{x}^{2} \mathrm{~d} x+\varepsilon \int_{0}^{\alpha} \int_{0}^{t} x g_{1} \\
& \cdot(t-s) u_{x}(x, t)\left[u_{x}(x, s)-u_{x}(x, t)\right] \mathrm{d} s \mathrm{~d} x+\varepsilon \int_{0}^{\alpha} \int_{0}^{t} x g_{2} \\
& \cdot(t-s) v_{x}(x, t)\left[v_{x}(x, s)-v_{x}(x, t)\right] \mathrm{d} s \mathrm{~d} x \\
& -\varepsilon \int_{0}^{\alpha} x u u_{t}\left|u_{t}\right|^{m-1} \mathrm{~d} x-\varepsilon \int_{0}^{\alpha} x v v_{t}\left|v_{t}\right|^{m-1} \mathrm{~d} x \\
& +\varepsilon 2(r+2) \int_{0}^{\alpha} x F(u, v) \mathrm{d} x .
\end{aligned}
$$

By using Young inequality and from $H(t)=-E(t)$,

$$
\begin{aligned}
\int_{0}^{\alpha} x F(u, v) \mathrm{d} x= & H(t)+\frac{1}{2} \int_{0}^{\alpha} x u_{t}^{2} \mathrm{~d} x+\frac{1}{2} \int_{0}^{\alpha} x v_{t}^{2} \mathrm{~d} x \\
& +\frac{1}{2}\left(1-\int_{0}^{t} g_{1}(s) \mathrm{d} s\right) \int_{0}^{\alpha} x u_{x}^{2} \mathrm{~d} x \\
& +\frac{1}{2}\left(1-\int_{0}^{t} g_{2}(s) \mathrm{d} s\right) \int_{0}^{\alpha} x v_{x}^{2} \mathrm{~d} x \\
& +\frac{1}{2}\left(g_{1} \circ u_{x}\right)(t)+\frac{1}{2}\left(g_{2} \circ v_{x}\right)(t),
\end{aligned}
$$

we obtain

$$
\begin{aligned}
L^{\prime}(t) \geq & (1-\sigma) H^{-\sigma}(t)\left[\left\|u_{t}\right\|_{L_{x}^{m+1}}^{m+1}+\left\|v_{t}\right\|_{L_{x}^{m+1}}^{m+1}\right] \\
& +\varepsilon(r+3) \int_{0}^{\alpha} x u_{t}^{2} \mathrm{~d} x+\varepsilon(r+3) \int_{0}^{\alpha} x v_{t}^{2} \mathrm{~d} x \\
& +\varepsilon\left[(r+1)-\left((r+1)+\frac{1}{4 \theta}\right) \int_{0}^{t} g_{1}(s) \mathrm{d} s\right] \int_{0}^{\alpha} x u_{x}^{2} \mathrm{~d} x \\
& +\varepsilon\left[(r+1)-\left((r+1)+\frac{1}{4 \theta}\right) \int_{0}^{t} g_{2}(s) \mathrm{d} s\right] \int_{0}^{\alpha} x v_{x}^{2} \mathrm{~d} x \\
& +\varepsilon(r-\theta+2)\left(g_{1} \circ u_{x}\right)(t)+\varepsilon(r-\theta+2)\left(g_{2} \circ v_{x}\right)(t)+\varepsilon 2(r+2) H(t) \\
& -\varepsilon \int_{0}^{\alpha} x u u_{t}\left|u_{t}\right|^{m-1} \mathrm{~d} x-\varepsilon \int_{0}^{\alpha} x v v_{t}\left|v_{t}\right|^{m-1} \mathrm{~d} x .
\end{aligned}
$$

$$
\begin{gathered}
\alpha_{3}=r-\theta+2>0 \Rightarrow r+2>\theta>0, \\
\alpha_{4}=r-\theta+2>0 \Rightarrow r+2>\theta>0, \\
\alpha_{1}=\left[(r+1)-\left((r+1)+\frac{1}{4 \theta}\right) \int_{0}^{t} g_{1}(s) \mathrm{d} s\right]>0, \\
\alpha_{2}=\left[(r+1)-\left((r+1)+\frac{1}{4 \theta}\right) \int_{0}^{t} g_{2}(s) \mathrm{d} s\right]>0 .
\end{gathered}
$$

From (34),

$$
\begin{aligned}
L^{\prime}(t) \geq & (1-\sigma) H^{-\sigma}(t)\left[\left\|u_{t}\right\|_{L_{x}+1}^{m+1}+\left\|v_{t}\right\|_{L_{x}^{m+1}}^{m+1}\right] \\
& +\varepsilon(r+3) \int_{0}^{\alpha} x u_{t}^{2} \mathrm{~d} x+\varepsilon(r+3) \int_{0}^{\alpha} x v_{t}^{2} \mathrm{~d} x+\varepsilon \alpha_{1} \int_{0}^{\alpha} x u_{x}^{2} \mathrm{~d} x \\
& +\varepsilon \alpha_{2} \int_{0}^{\alpha} x v_{x}^{2} \mathrm{~d} x+\varepsilon \alpha_{3}\left(g_{1} \circ u_{x}\right)(t)+\varepsilon \alpha_{4}\left(g_{2} \circ v_{x}\right)(t) \\
& +\varepsilon 2(r+2) H(t)-\varepsilon \int_{0}^{\alpha} x u u_{t}\left|u_{t}\right|^{m-1} \mathrm{~d} x-\varepsilon \int_{0}^{\alpha} x v v_{t}\left|v_{t}\right|^{m-1} \mathrm{~d} x, \\
H(t)= & E(t)=-\frac{1}{2} \int_{0}^{\alpha} x u_{t}^{2} \mathrm{~d} x-\frac{1}{2} \int_{0}^{\alpha} x v_{t}^{2} \mathrm{~d} x \\
& -\frac{1}{2}\left(1-\int_{0}^{t} g_{1}(s) \mathrm{d} s\right) \int_{0}^{\alpha} x u_{x}^{2} \mathrm{~d} x-\frac{1}{2}\left(1-\int_{0}^{t} g_{2}(s) \mathrm{d} s\right) \int_{0}^{\alpha} x v_{x}^{2} \mathrm{~d} x \\
& -\frac{1}{2}\left(g_{1} \circ u_{x}\right)(t)-\frac{1}{2}\left(g_{2} \circ v_{x}\right)(t)+\int_{0}^{\alpha} x F(u, v) \mathrm{d} x,
\end{aligned}
$$

and for $a_{5}<\min \left\{\alpha_{1}, \alpha_{2}, \alpha_{3}, \alpha_{4}, 2(r+2)\right\}$,

$$
\begin{aligned}
\varepsilon 2(r+2) H(t)= & \varepsilon\left(a_{5}+\left(2(r+2)-a_{5}\right)\right) H(t) \\
= & \varepsilon a_{5} H(t)+\varepsilon\left(2(r+2)-a_{5}\right) H(t) \\
= & -\frac{\varepsilon}{2} a_{5} \int_{0}^{\alpha} x u_{t}^{2} \mathrm{~d} x-\frac{\varepsilon}{2} a_{5} \int_{0}^{\alpha} x v_{t}^{2} \mathrm{~d} x \\
& -\frac{\varepsilon}{2} a_{5}\left(1-\int_{0}^{t} g_{1}(s) \mathrm{d} s\right) \int_{0}^{\alpha} x u_{x}^{2} \mathrm{~d} x \\
& -\frac{\varepsilon}{2} a_{5}\left(1-\int_{0}^{t} g_{2}(s) \mathrm{d} s\right) \int_{0}^{\alpha} x v_{x}^{2} \mathrm{~d} x \\
& -\frac{\varepsilon}{2} a_{5}\left(g_{1} \circ u_{x}\right)(t)-\frac{\varepsilon}{2} a_{5}\left(g_{2} \circ v_{x}\right)(t) \\
& +\varepsilon a_{5} \int_{0}^{\alpha} x F(u, v) \mathrm{d} x+\varepsilon\left(\left(2(r+2)-a_{5}\right)\right) H(t) .
\end{aligned}
$$

To estimate the last term in (36), we apply the threeparameter Young inequality: $a, b \geq 0,(1 / r)+(1 / q)=1$ $, a b \leq\left(\delta^{r} / r\right) a^{r}+\left(\delta^{-q} b^{q} / q\right), \forall \delta>0$. We take

$$
r=m+1 v e q=\frac{m+1}{m}
$$

in this case:

$$
-\varepsilon \int_{0}^{\alpha} x u u_{t}\left|u_{t}\right|^{m-1} \mathrm{~d} x \geq-\varepsilon \frac{\delta_{1}^{m+1}}{m+1}\|u\|_{L_{x}^{m+1}}^{m+1}-\varepsilon \frac{m}{m+1} \delta_{1}^{-((m+1) / m)}\left\|u_{t}\right\|_{L_{x}^{m+1}}^{m+1}
$$

where 
Similarly

$$
\begin{aligned}
-\varepsilon \int_{0}^{\alpha} x v v_{t}\left|v_{t}\right|^{m-1} \mathrm{~d} x \geq & -\varepsilon \frac{\delta_{2}^{m+1}}{m+1}\|v\|_{L_{x}^{m+1}}^{m+1} \\
& -\varepsilon \frac{m}{m+1} \delta_{2}^{-((m+1) / m)}\left\|v_{t}\right\|_{L_{x}^{m+1}}^{m+1}
\end{aligned}
$$

Substituting (38), (40), and (41) into (36), by organizing, we obtain

$$
\begin{aligned}
L^{\prime}(t) \geq & {\left[(1-\sigma) H^{-\sigma}(t)-\frac{m}{\mathrm{~m}+1} \varepsilon \delta_{1}^{-((m+1) / m)}\right]\left\|u_{t}\right\|_{L_{x}^{m+1}}^{m+1} } \\
& +\left[(1-\sigma) H^{-\sigma}(t)-\frac{m}{m+1} \varepsilon \delta_{2}^{-((m+1) / m)}\right]\left\|v_{t}\right\|_{L_{x}^{m+1}}^{m+1} \\
& +\varepsilon\left((r+3)-\frac{a_{5}}{2}\right) \int_{0}^{\alpha} x u_{t}^{2} \mathrm{~d} x+\varepsilon\left((r+3)-\frac{a_{5}}{2}\right) \int_{0}^{\alpha} x v_{t}^{2} \mathrm{~d} x \\
& +\varepsilon\left(\alpha_{1}-\frac{a_{5}}{2}\left(1-\int_{0}^{t} g_{1}(s) \mathrm{d} s\right)\right) \int_{0}^{\alpha} x u_{x}^{2} \mathrm{~d} x \\
& +\varepsilon\left(\alpha_{2}-\frac{a_{5}}{2}\left(1-\int_{0}^{t} g_{2}(s) \mathrm{d} s\right)\right) \int_{0}^{\alpha} x v_{x}^{2} \mathrm{~d} x \\
& +\varepsilon\left(\alpha_{3}-\frac{a_{5}}{2}\right)\left(g_{1} \circ u_{x}\right)(t)+\varepsilon\left(\alpha_{4}-\frac{a_{5}}{2}\right)\left(g_{2} \circ v_{x}\right)(t) \\
& +\varepsilon a_{5} \int_{0}^{\alpha} x F(u, v) \mathrm{d} x+\varepsilon\left(\left(2(r+2)-a_{5}\right)\right) H(t) \\
& -\varepsilon \frac{\delta_{1}^{m+1}}{m+1}\|u\|_{L_{x}^{m+1}}^{m+1}-\varepsilon \frac{\delta_{2}^{m+1}}{m+1}\|v\|_{L_{x}^{m+1}}^{m+1} .
\end{aligned}
$$

Since integration in estimate (40) and (41) is performed over the space, the parameter $\delta_{1}$ and $\delta_{2}$ can be a function of time; we get them as follows:

$$
\begin{gathered}
\delta_{1}^{-((m+1) / m)}=k_{1} H^{-\sigma}(t) \Rightarrow \delta_{1}^{m+1}=k_{1}^{-m} H^{\sigma m}(t), \\
\delta_{2}^{-((m+1) / m)}=k_{2} H^{-\sigma}(t) \Rightarrow \delta_{2}^{m+1}=k_{2}^{-m} H^{\sigma m}(t),
\end{gathered}
$$

where $k_{1}>0$ and $k_{2}>0$ are sufficiently large constants to be specified further. By using (43) and (44) in (42), we have

$$
\begin{aligned}
L^{\prime}(t) \geq & \left((1-\sigma)-\frac{m}{m+1} \varepsilon k_{1}\right) H^{-\sigma}(t)\left\|u_{t}\right\|_{L_{x}^{m+1}}^{m+1} \\
& +\left((1-\sigma)-\frac{m}{m+1} \varepsilon k_{2}\right) H^{-\sigma}(t)\left\|v_{t}\right\|_{L_{x}^{m+1}}^{m+1} \\
& +\varepsilon\left((r+3)-\frac{a_{5}}{2}\right) \int_{0}^{\alpha} x u_{t}^{2} \mathrm{~d} x+\varepsilon\left((r+3)-\frac{a_{5}}{2}\right) \int_{0}^{\alpha} x v_{t}^{2} \mathrm{~d} x \\
& +\varepsilon\left(\alpha_{1}-\frac{a_{5}}{2}\left(1-\int_{0}^{t} g_{1}(s) \mathrm{d} s\right)\right) \int_{0}^{\alpha} x u_{x}^{2} \mathrm{~d} x \\
& +\varepsilon\left(\alpha_{2}-\frac{a_{5}}{2}\left(1-\int_{0}^{t} g_{2}(s) \mathrm{d} s\right)\right) \int_{0}^{\alpha} x v_{x}^{2} \mathrm{~d} x \\
& +\varepsilon\left(\alpha_{3}-\frac{a_{5}}{2}\right)\left(g_{1} \circ u_{x}\right)(t)+\varepsilon\left(\alpha_{4}-\frac{a_{5}}{2}\right)\left(g_{2} \circ v_{x}\right)(t) \\
& +\varepsilon a_{5} \int_{0}^{\alpha} x F(u, v) \mathrm{d} x+\varepsilon\left(\left(2(r+2)-a_{5}\right)\right) H(t) \\
& -\frac{\varepsilon k_{1}^{-m}}{m+1} H^{\sigma m}(t)\|u\|_{L_{x}^{m+1}}^{m+1}-\frac{\varepsilon k_{2}^{-m}}{m+1} H^{\sigma m}(t)\|v\|_{L_{x}^{m+1}}^{m+1} .
\end{aligned}
$$

To estimate the last two terms in (45), we use (29); then, $\frac{\varepsilon k_{1}^{-m}}{m+1}(H(t))^{\sigma m}\|u\|_{L_{x}^{m+1}}^{m+1} \leq \frac{\varepsilon k_{1}^{-m} c_{2}^{\sigma m}}{m+1}\left[\|u\|_{L_{x}^{2(r+2)}}^{2(r+2)}+\|v\|_{L_{x}^{2(r+2)}}^{2(r+2)}\right]^{\sigma m}\|u\|_{L_{x}^{m+1}}^{m+1}$.

On the other hand, since $r>\max \{m, m\}$ from $L_{x}^{2(r+2)^{\circ}}$ $L_{x}^{m+1}$,

$$
\|u\|_{L_{x}^{m+1}}^{m+1} \leq C\|u\|_{L_{x}^{2(r+2)}}^{m+1} \leq C\left[\|u\|_{L_{x}^{2(r+2)}}+\|v\|_{L_{x}^{2(r+2)}}\right]^{m+1} .
$$

Substituting (47) into (46),

$$
\begin{aligned}
\frac{\varepsilon k_{1}^{-m}}{m+1}(H(t))^{\sigma m}\|u\|_{L_{x}^{m+1}}^{m+1} \leq & \frac{\varepsilon k_{1}^{-m} c_{2}^{\sigma m} C}{m+1}\left[\|u\|_{L_{x}^{2(r+2)}}^{2(r+2)}+\|v\|_{L_{x}^{2(r+2)}}^{2(r+2)}\right]^{\sigma m} \\
& \cdot\left[\|u\|_{L_{x}^{2(r+2)}}+\|v\|_{L_{x}^{2(r+2)}}\right]^{m+1} .
\end{aligned}
$$

By using

$$
\begin{gathered}
a, b \geq 0, \\
1 \leq p<\infty, \\
a^{p}+b^{p} \leq(a+b)^{p},
\end{gathered}
$$

we can estimate the following:

$$
\left[\|u\|_{L_{x}^{2(r+2)}}^{2(r+2)}+\|v\|_{L_{x}^{2(r+2)}}^{2(r+2)}\right] \leq\left[\|u\|_{L_{x}^{2(r+2)}}+\|v\|_{L_{x}^{2(r+2)}}\right]^{2(r+2)} .
$$

Consequently, we have

$\frac{\varepsilon k_{1}^{-m}}{m+1}(H(t))^{\sigma m}\|u\|_{L_{x}^{m+1}}^{m+1} \leq \frac{\varepsilon k_{1}^{-m} c_{2}^{\sigma m} C}{m+1}\left[\|u\|_{L_{x}^{2(r+2)}}+\|v\|_{L_{x}^{2(r+2)}}\right]^{2(r+2) \sigma m+m+1}$.

Similarly

$\frac{\varepsilon k_{2}^{-m}}{m+1}(H(t))^{\sigma m}\|v\|_{L_{x}^{m+1}}^{m+1} \leq \frac{\varepsilon k_{2}^{-m} c_{2}^{\sigma m} C}{m+1}\left[\|u\|_{L_{x}^{2(r+2)}}+\|v\|_{L_{x}^{2(r+2)}}\right]^{2(r+2) \sigma m+m+1}$.

By using (51) and (52),

$a, b \geq 0, \quad 1 \leq p<\infty,(a+b)^{p} \leq c\left(a^{p}+b^{p}\right), \quad\left(c=2^{p-1}\right)$,

for $\cdot C=C^{\prime}$; we have

$$
\begin{aligned}
& \frac{\varepsilon k_{1}^{-m}}{m+1}(H(t))^{\sigma m}\|u\|_{L_{x}^{m+1}}^{m+1} \leq \frac{\varepsilon k_{1}^{-m} c_{2}^{\sigma m} C^{\prime}}{m+1}\left[\|u\|_{L_{x}^{2(r+2)}}^{2(r+2) \sigma m+m+1}+\|v\|_{L_{x}^{2(r+2)}}^{2(r+2) \sigma m+m+1}\right], \\
& \frac{\varepsilon k_{2}^{-m}}{m+1}(H(t))^{\sigma m}\|v\|_{L_{x}^{m+1}}^{m+1} \leq \frac{\varepsilon k_{2}^{-m} c_{2}^{\sigma m} C^{\prime}}{m+1}\left[\|u\|_{L_{x}^{2(r+2)}}^{2(r+2) \sigma m+m+1}+\|v\|_{L_{x}^{2(r+2)}}^{2(r+2) \sigma m+m+1}\right] .
\end{aligned}
$$


From (31),

$$
\begin{gathered}
2(r+2) \sigma m+m+1 \leq 2(r+2)\left(\frac{2(r+2)-m}{2 m(r+2)}\right) m+m+1=2(r+2)+1, \\
r \geq-1 \\
2(r+2) \geq 2 \\
2(r+2) \sigma m+m+1 \geq 2 .
\end{gathered}
$$

From here,

$$
2 \leq 2(r+2) \sigma m+m+1 \leq 2(r+2)+1 \text {. }
$$

Thus, by applying (23), we obtain

$$
\begin{gathered}
\|u\|_{L_{x}^{2(r+2)}}^{2(r+2) \sigma m+m+1} \leq\left\|u_{x}\right\|_{H}^{2}+\|u\|_{L_{x}^{2(r+2)}}^{2(r+2)}, \\
\|v\|_{L_{x}^{2(r+2)}}^{2(r+2) \sigma m+m+1} \leq\left\|v_{x}\right\|_{H}^{2}+\|v\|_{L_{x}^{2(r+2)}}^{2(r+2)} .
\end{gathered}
$$
case,

Substituting these inequalities in (54) and (55), in this

$$
\frac{\varepsilon k_{1}^{-m}}{m+1}(H(t))^{\sigma m}\|u\|_{L_{x}^{m+1}}^{m+1} \leq \frac{\varepsilon k_{1}^{-m} c_{2}^{\sigma m} C^{\prime}}{m+1}\left[\left\|u_{x}\right\|_{H}^{2}+\|u\|_{L_{x}^{(2+2)}}^{2(r+2)}+\left\|v_{x}\right\|_{H}^{2}+\|v\|_{L_{x}^{2(r+2)}}^{2(r+2)}\right],
$$

$\frac{\varepsilon k_{2}^{-m}}{m+1}(H(t))^{\sigma m}\|v\|_{L_{x}^{m+1}}^{m+1} \leq \frac{\varepsilon k_{2}^{-m} c_{2}^{\sigma m} C^{\prime}}{m+1}\left[\left\|u_{x}\right\|_{H}^{2}+\|u\|_{L_{x}^{(2 x+2)}}^{2(r+2)}+\left\|v_{x}\right\|_{H}^{2}+\|v\|_{L_{x}^{2(r+2)}}^{2(r+2)}\right]$.

With the combination of (59) and (60), we obtain

$$
\begin{aligned}
& -\frac{\varepsilon k_{1}^{-m}}{m+1} H^{\sigma m}(t)\|u\|_{L_{x}^{m+1}}^{m+1}-\frac{\varepsilon k_{2}^{-m}}{m+1} H^{\sigma m}(t)\|v\|_{L_{x}^{m+1}}^{m+1} \\
& \geq\left[-\frac{\varepsilon k_{1}^{-m} c_{2}^{\sigma m} C^{\prime}}{m+1}-\frac{\varepsilon k_{2}^{-m} c_{2}^{\sigma m} C^{\prime}}{m+1}\right]\left(\|u\|_{L_{x}^{2(r+2)}}^{2(r+2)}+\|v\|_{L_{x}^{2(r+2)}}^{2(r+2)}\right) \\
& +\left[-\frac{\varepsilon k_{1}^{-m} c_{2}^{\sigma m} C^{\prime}}{m+1}-\frac{\varepsilon k_{2}^{-m} c_{2}^{\sigma m} C^{\prime}}{m+1}\right]\left(\left\|u_{x}\right\|_{H}^{2}+\left\|v_{x}\right\|_{H}^{2}\right) .
\end{aligned}
$$

Finally,

$$
\begin{gathered}
\left\|u_{x}\right\|_{H}^{2}=\int_{0}^{\alpha} x u_{x}^{2} \mathrm{~d} x,\left\|v_{x}\right\|_{H}^{2}=\int_{0}^{\alpha} x v_{x}^{2} \mathrm{~d} x, \\
\|u\|_{L_{x}^{2(r+2)}}^{2(r+2)}=\int_{0}^{\alpha} x|u|^{2(r+2)} \mathrm{d} x,\|v\|_{L_{x}^{2(r+2)}}^{2(r+2)}=\int_{0}^{\alpha} x|v|^{2(r+2)} \mathrm{d} x, \\
c^{\prime} \cdot\left(\int_{0}^{\alpha} x|u|^{2(r+2)} \mathrm{d} x+\int_{0}^{\alpha} x|v|^{2(r+2)} \mathrm{d} x\right) \leq \int_{0}^{\alpha} x F(u, v) \mathrm{d} x,\left(\frac{c_{0}}{2(r+2)}=c^{\prime}\right),
\end{gathered}
$$

and by considering (61), thus by organizing (45), we have

$$
\begin{aligned}
L^{\prime}(t) \geq & \left((1-\sigma)-\frac{m}{m+1} \varepsilon k_{1}\right) H^{-\sigma}(t)\left\|u_{t}\right\|_{L_{x}^{m+1}}^{m+1} \\
& +\left((1-\sigma)-\frac{m}{m+1} \varepsilon k_{2}\right) H^{-\sigma}(t)\left\|v_{t}\right\|_{L_{x}^{m+1}}^{m+1} \\
& +\varepsilon\left((r+3)-\frac{a_{5}}{2}\right) \int_{0}^{\alpha} x u_{t}^{2} \mathrm{~d} x+\varepsilon\left((r+3)-\frac{a_{5}}{2}\right) \int_{0}^{\alpha} x v_{t}^{2} \mathrm{~d} x \\
& +\varepsilon\left[\left(\alpha_{1}-\frac{a_{5}}{2}\left(1-\int_{0}^{t} g_{1}(s) \mathrm{d} s\right)\right)-\frac{k_{1}^{-m} c_{2}^{\sigma m} C^{\prime}}{m+1}-\frac{k_{2}^{-m} c_{2}^{\sigma m} C^{\prime}}{m+1}\right] \\
& \cdot \int_{0}^{\alpha} x u_{x}^{2} \mathrm{~d} x+\varepsilon\left[\left(\alpha_{2}-\frac{a_{5}}{2}\left(1-\int_{0}^{t} g_{2}(s) \mathrm{d} s\right)\right)-\frac{k_{1}^{-m} c_{2}^{\sigma m} C^{\prime}}{m+1}-\frac{k_{2}^{-m} c_{2}^{\sigma m} C^{\prime}}{m+1}\right] \\
& \cdot \int_{0}^{\alpha} x v_{x}^{2} \mathrm{~d} x+\varepsilon\left[c^{\prime} a_{5}-\frac{k_{1}^{-m} c_{2}^{\sigma m} C^{\prime}}{m+1}-\frac{k_{2}^{-m} c_{2}^{\sigma m} C^{\prime}}{m+1}\right] \int_{0}^{\alpha} x|u|^{2(r+2)} \mathrm{d} x \\
& +\varepsilon\left[c^{\prime} a_{5}-\frac{k_{1}^{-m} c_{2}^{\sigma m} C^{\prime}}{m+1}-\frac{\left.k_{2}^{-m} c_{2}^{\sigma m} C^{\prime}\right]}{m+1}\right] \int_{0}^{\alpha} x|v|^{2(r+2)} \mathrm{d} x \\
& +\varepsilon\left[\alpha_{3}-\frac{a_{5}}{2}\right]\left(g_{1} \circ u_{x}\right)(t)+\varepsilon\left[\alpha_{4}-\frac{a_{5}}{2}\right]\left(g_{2} \circ u_{x}\right)(t)+\varepsilon\left[2(r+2)-a_{5}\right] H(t),
\end{aligned}
$$

which introduce the constant

$$
\begin{aligned}
& \gamma=\varepsilon \cdot \min \left\{(r+3)-\frac{a_{5}}{2},\left[\left(\alpha_{1}-\frac{a_{5}}{2}\left(1-\int_{0}^{t} g_{1}(s) \mathrm{d} s\right)\right)-\frac{k_{1}^{-m} c_{2}^{\sigma m} C^{\prime}}{m+1}-\frac{k_{2}^{-m} c_{2}^{\sigma m} C^{\prime}}{m+1}\right],\right. \\
& {\left[\left(\alpha_{2}-\frac{a_{5}}{2}\left(1-\int_{0}^{t} g_{2}(s) \mathrm{d} s\right)\right)-\frac{k_{1}^{-m} c_{2}^{\sigma m} C^{\prime}}{m+1}-\frac{k_{2}^{-m} c_{2}^{\sigma m} C^{\prime}}{m+1}\right], } \\
& {\left.\left[c^{\prime} a_{5}-\frac{k_{1}^{-m} c_{2}^{\sigma m} C^{\prime}}{m+1}-\frac{k_{2}^{-m} c_{2}^{\sigma m} C^{\prime}}{m+1}\right],\left[\alpha_{3}-\frac{a_{5}}{2}\right],\left[\alpha_{4}-\frac{a_{5}}{2}\right],\left[2(r+2)-a_{5}\right]\right\} . }
\end{aligned}
$$

Taking sufficiently large $k_{1}>0$ and $k_{2}>0$ for the positive constant $\gamma$, we simplify (63)

$$
\begin{aligned}
L^{\prime}(t) \geq & \left((1-\sigma)-\frac{m}{m+1} \varepsilon k_{1}\right) H^{-\sigma}(t)\left\|u_{t}\right\|_{L_{x}^{m+1}}^{m+1} \\
& +\left((1-\sigma)-\frac{m}{m+1} \varepsilon k_{2}\right) H^{-\sigma}(t)\left\|v_{t}\right\|_{L_{x}^{m+1}}^{m+1} \\
& +\varepsilon \gamma\left[\int_{0}^{\alpha} x u_{t}^{2} \mathrm{~d} x+\int_{0}^{\alpha} x v_{t}^{2} \mathrm{~d} x+\int_{0}^{\alpha} x u_{x}^{2} \mathrm{~d} x+\int_{0}^{\alpha} x v_{x}^{2} \mathrm{~d} x\right. \\
& +\int_{0}^{\alpha} x|u|^{2(r+2)} \mathrm{d} x+\int_{0}^{\alpha} x|\mathrm{v}|^{2(r+2)} \mathrm{d} x+\left(g_{1} \circ u_{x}\right)(t) \\
& \left.+\left(g_{2} \circ v_{x}\right)(t)+H(t)\right] .
\end{aligned}
$$

For fixed $k_{1}>0, k_{2}>0$, and $\gamma>0$, we choose $\varepsilon>0$ so small that the following inequality holds:

$$
\begin{aligned}
& \left((1-\sigma)-\frac{m}{m+1} \varepsilon k_{1}\right) \geq 0, \\
& \left((1-\sigma)-\frac{m}{m+1} \varepsilon k_{2}\right) \geq 0 .
\end{aligned}
$$

Moreover, we assume that the initial data satisfy the estimate

$$
L(0)=H^{1-\sigma}(0)+\varepsilon\left(\int_{0}^{\alpha} x u_{0} u_{1} \mathrm{~d} x+\int_{0}^{\alpha} x v_{0} v_{1} \mathrm{~d} x\right)>0 .
$$


Then, from (65), we obtain the following inequality:

$$
\begin{aligned}
L^{\prime}(t) \geq & \varepsilon \gamma\left[\int_{0}^{\alpha} x u_{t}^{2} \mathrm{~d} x+\int_{0}^{\alpha} x v_{t}^{2} \mathrm{~d} x+\int_{0}^{\alpha} x u_{x}^{2} \mathrm{~d} x+\int_{0}^{\alpha} x v_{x}^{2} \mathrm{~d} x\right. \\
& +\int_{0}^{\alpha} \mathrm{x}|u|^{2(r+2)} \mathrm{d} x+\int_{0}^{\alpha} x|v|^{2(r+2)} \mathrm{d} x+\left(g_{1} \circ u_{x}\right)(t) \\
& \left.+\left(g_{2} \circ v_{x}\right)(t)+H(t)\right] .
\end{aligned}
$$

On the other hand, in Equation (30), we take the $1 /(1-\sigma) \cdot$ powerof each side

$$
[L(t)]^{1 /(1-\sigma)}=\left[H^{1-\sigma}(t)+\varepsilon\left(\int_{0}^{\alpha} x u u_{t} \mathrm{~d} x+\int_{0}^{\alpha} x v v_{t} \mathrm{~d} x\right)\right]^{1 /(1-\sigma)}
$$

Twice by applying the following inequality to (69)

$$
a, b \geq 0,1 \leq p<\infty,(a+b)^{p} \leq 2^{p-1}\left(a^{p}+b^{p}\right),
$$

we have

$$
\begin{aligned}
{[L(t)]^{1 /(1-\sigma)} } & \leq 2^{\sigma /(1-\sigma)}\left[H(t)+\varepsilon^{1 /(1-\sigma)}|| \int_{0}^{\alpha} x u u_{t} \mathrm{~d} x+\left.\int_{0}^{\alpha} x v v_{t} \mathrm{~d} x\right|^{1 /(1-\sigma)}\right] \\
& \leq 2^{\sigma /(1-\sigma)}\left[H(t)+\varepsilon^{1 /(1-\sigma)} 2^{\sigma /(1-\sigma)}\left(\left|\int_{0}^{\alpha} x u u_{t} \mathrm{~d} x\right|^{1 /(1-\sigma)}+\left|\int_{0}^{\alpha} x v v_{t} \mathrm{~d} x\right|^{1 /(1-\sigma)}\right)\right] \\
& \leq C\left[H(t)+\left|\int_{0}^{\alpha} x u u_{t} \mathrm{~d} x\right|^{1 /(1-\sigma)}+\left|\int_{0}^{\alpha} x v v_{t} \mathrm{~d} x\right|^{1 /(1-\sigma)}\right]
\end{aligned}
$$

where $C>0$. Now, to estimate the last two terms in (71), we, respectively, apply Holder inequality, $L_{x}^{2(r+2)^{\circ}} L_{x}^{H}$, and Young inequality; thus,

$$
\begin{aligned}
\left|\int_{0}^{\alpha} x u u_{t} \mathrm{~d} x\right|^{1 /(1-\sigma)} & \leq\|u\|_{H}^{1 /(1-\sigma)}\left\|u_{t}\right\|_{H}^{1 /(1-\sigma)} \leq C\|u\|_{L_{x}^{2(r+2)}}^{1 /(1-\sigma)}\left\|u_{t}\right\|_{H}^{1 /(1-\sigma)} \\
& \leq C\left(\|u\|_{L_{x}^{2(r+2)}}^{\theta /(1-\sigma)}+\left\|u_{t}\right\|_{H}^{\mu /(1-\sigma)}\right) .
\end{aligned}
$$

Similarly,

$$
\left|\int_{0}^{\alpha} x v v_{t} \mathrm{~d} x\right|^{1 /(1-\sigma)} \leq C\left(\|v\|_{L_{x}^{2(r+2)}}^{\theta /(1-\sigma)}+\left\|v_{t}\right\|_{H}^{\mu /(1-\sigma)}\right)
$$

where $(1 / \theta)+(1 / \mu)=1$. In these inequalities by collecting side by side, we obtain

$$
\begin{aligned}
& \left|\int_{0}^{\alpha} x u u_{t} \mathrm{~d} x\right|^{1 /(1-\sigma)}+\left|\int_{0}^{\alpha} x v v_{t} \mathrm{~d} x\right|^{1 /(1-\sigma)} \\
& \quad \leq C\left(\|u\|_{L_{x}^{2(r+2)}}^{\theta /(1-\sigma)}+\left\|u_{t}\right\|_{H}^{\mu /(1-\sigma)}+\|v\|_{L_{x}^{2(r+2)}}^{\theta /(1-\sigma)}+\left\|v_{t}\right\|_{H}^{\mu /(1-\sigma)}\right) .
\end{aligned}
$$

We choose $\mu=2(1-\sigma)$, to get

$$
\theta=\frac{2(1-\sigma)}{1-2 \sigma} \leq 2(r+2)
$$

then

$$
\begin{aligned}
& \left|\int_{0}^{\alpha} x u u_{t} \mathrm{~d} x\right|^{1 /(1-\sigma)}+\left|\int_{0}^{\alpha} x v v_{t} \mathrm{~d} x\right|^{1 /(1-\sigma)} \\
& \quad \leq C\left(\|u\|_{L_{x}^{2(r+2)}}^{2 /(1-2 \sigma)}+\left\|u_{t}\right\|_{H}^{2}+\|v\|_{L_{x}^{2(r+2)}}^{2 /(1-2 \sigma)}+\left\|v_{t}\right\|_{H}^{2}\right) .
\end{aligned}
$$

By applying (23), we can write

$$
\begin{gathered}
\|u\|_{L_{x}^{2(r+2)}}^{2 /(1-2 \sigma)} \leq C\left(\left\|u_{x}\right\|_{H}^{2}+\|u\|_{L_{x}^{2(r+2)}}^{2(r+2)}\right), \\
\|v\|_{L_{x}^{2(r+2)}}^{2 /(1-2 \sigma)} \leq C\left(\left\|v_{x}\right\|_{H}^{2}+\|v\|_{L_{x}^{2(r+2)}}^{2(r+2)}\right) .
\end{gathered}
$$

From here, we obtain

$$
\begin{aligned}
& \left|\int_{0}^{\alpha} x u u_{t} \mathrm{~d} x\right|^{1 /(1-\sigma)}+\left|\int_{0}^{\alpha} x v v_{t} \mathrm{~d} x\right|^{1 /(1-\sigma)} \\
& \quad \leq C\left(\left\|u_{t}\right\|_{H}^{2}+\left\|v_{t}\right\|_{H}^{2}+\left\|u_{x}\right\|_{H}^{2}+\left\|v_{x}\right\|_{H}^{2}+\|u\|_{L_{x}^{2(r+2)}}^{2(r+2)}+\|v\|_{L_{x}^{2(r+2)}}^{2(r+2)}\right) .
\end{aligned}
$$

Thus, by considering (78) and the following in (71),

$$
\begin{gathered}
\left\|u_{t}\right\|_{H}^{2}=\int_{0}^{\alpha} x u_{t}^{2} \mathrm{~d} x,\left\|v_{t}\right\|_{H}^{2}=\int_{0}^{\alpha} x v_{t}^{2} \mathrm{~d} x, \\
\left\|u_{x}\right\|_{H}^{2}=\int_{0}^{\alpha} x u_{x}^{2} \mathrm{~d} x,\left\|v_{x}\right\|_{H}^{2}=\int_{0}^{\alpha} x v_{x}^{2} \mathrm{~d} x, \\
\|u\|_{L_{x}^{2(r+2)}}^{2(r+2)}=\int_{0}^{\alpha} x|u|^{2(r+2)} \mathrm{d} x,\|v\|_{L_{x}^{2(r+2)}}^{2(r+2)}=\int_{0}^{\alpha} x|v|^{2(r+2)} \mathrm{d} x, \\
\left(g_{1} \circ u_{x}\right)(t) \geq 0,\left(g_{2} \circ v_{x}\right)(t) \geq 0,
\end{gathered}
$$

we obtain

$$
\begin{aligned}
{[L(t)]^{1 /(1-\sigma)} \leq } & C\left[H(t)+\int_{0}^{\alpha} x u_{t}^{2} \mathrm{~d} x+\int_{0}^{\alpha} x v_{t}^{2} \mathrm{~d} x+\int_{0}^{\alpha} x u_{x}^{2} \mathrm{~d} x\right. \\
& +\int_{0}^{\alpha} x v_{x}^{2} \mathrm{~d} x+\int_{0}^{\alpha} x|u|^{2(r+2)} \mathrm{d} x+\int_{0}^{\alpha} x|v|^{2(r+2)} \mathrm{d} x \\
& \left.+\left(g_{1} \circ u_{x}\right)(t)+\left(g_{2} \circ v_{x}\right)(t)\right] .
\end{aligned}
$$

Finally, by combining (68) and (80), we obtain the following ordinary differential inequality:

$$
L^{\prime}(t) \geq \lambda L^{1 /(1-\sigma)}(t) \forall t \geq 0
$$

obviously, where $\lambda>0$ is a constant depending only $C$, $\varepsilon$, and 
$\gamma$. This differential inequality integration over $(0, t)$ gives

$$
L^{1 /(1-\sigma)}(t) \geq \frac{1}{L^{-\sigma /(1-\sigma)}(0)-\lambda(\sigma /(1-\sigma)) t},
$$

where we choose

$$
t \leq T^{*}=\frac{1-\sigma}{\lambda \sigma L^{\sigma /(1-\sigma)}(0)} .
$$

Hence,

$$
\lim _{t \longrightarrow T^{*-}} L(t) \longrightarrow \infty
$$

\section{Conclusions}

The purpose of this paper is to study the explosion result of the solution of the system of nonlocal singular viscoelastic with damping and source terms on general case. This current study is a general case of the previous work of Boulaaras in ([5]). In the next work, we will try to obtain the same result for the two-dimensional problem that allows a reasonable description of the phenomenon occurring in a threedimensional domain. Then, we will try to prove uniqueness results of the weak solution.

\section{Data Availability}

No data were used to support the study.

\section{Conflicts of Interest}

The authors declare that they have no conflicts of interest.

\section{References}

[1] A. Rouabhia, A. Chikh, A. Bousahla et al., "Physical stability response of a SLGS resting on viscoelastic medium using nonlocal integral first-order theory," Steel and Composite Structures, vol. 37, no. 6, pp. 695-709, 2020.

[2] M. S. H. Al-Furjan, M. Habibi, J. Ni, D. . Jung, and A. Tounsi, "Frequency simulation of viscoelastic multi-phase reinforced fully symmetric systems," Engineering with Computers, 2020, In press.

[3] J. Ball, "Remarks on blow-up and nonexistence theorems for nonlinear evolution equations," Quarterly Journal of Mathematics, vol. 28, no. 4, pp. 473-486, 1977.

[4] S. Berrimi and S. Messaoudi, "Existence and decay of solutions of a viscoelastic equation with a nonlinear source," Nonlinear Analysis, vol. 64, no. 10, pp. 2314-2331, 2006.

[5] S. Boulaaras, R. Guefaifia, and N. Mezouar, "Global existence and decay for a system of two singular one-dimensional nonlinear viscoelastic equations with general source terms," Applicable Analysis, pp. 1-25, 2020, In press.

[6] S. Boulaaras, R. Guefaifia, N. Mezouar, and A. M. Alghamdi, "Global existence and decay for a system of two singular nonlinear viscoelastic equations with general source and localized frictional damping terms," Journal of Function Spaces, vol. 2020, Article ID 5085101, 15 pages, 2020.
[7] S. Boulaaras and Y. Bouizem, "Blow up of solutions for a nonlinear viscoelastic system with general source term," Quaestiones Mathematicae, pp. 1-11, 2020, In press.

[8] M. M. Cavalcanti, V. N. Domingos Cavalcanti, and J. Ferreira, "Existence and uniform decay for a non-linear viscoelastic equation with strong damping," Mathematical Methods in the Applied Sciences, vol. 24, no. 14, pp. 1043-1053, 2001.

[9] Y.S. Choi and K. Y. Chan, "A parabolic equation with nonlocal boundary conditions arising from electrochemistry," Nonlinear Analysis: Theory, Methods \& Applications, vol. 18, no. 4, pp. 317-331, 1992.

[10] S. Gala and M. A. Ragusa, "Logarithmically improved regularity criterion for the Boussinesq equations in Besov spaces with negative indices," Applicable Analysis, vol. 95, no. 6, pp. 12711279, 2016.

[11] S. Gala, Q. Liu, and M. A. Ragusa, "A new regularity criterion for the nematic liquid crystal flows," Applicable Analysis, vol. 91, no. 9, pp. 1741-1747, 2012.

[12] M. Kafini and S. A. Messaoudi, "A blow-up result in a Cauchy viscoelastic problem," Applied Mathematics Letters, vol. 21, no. 6, pp. 549-553, 2008.

[13] L. Guo, Z. Yuan, and G. Lin, "Blow up and global existence for a nonlinear viscoelastic wave equation with strong damping and nonlinear damping and source terms," Applied Mathematics, vol. 6, no. 5, pp. 806-816, 2015.

[14] M. R. Li and L. Y. Tsai, "Existence and nonexistence of global solutions of some system of semilinear wave equations," Nonlinear Analysis: Theory, Methods \& Applications, vol. 54, no. 8, pp. 1397-1415, 2003.

[15] S. Mesloub, "A nonlinear nonlocal mixed problem for a second order pseudoparabolic equation," Journal of Mathematical Analysis and Applications, vol. 316, no. 1, pp. 189-209, 2006.

[16] S. Mesloub and A. Bouziani, "Mixed problem with a weighted integral condition for a parabolic equation with the Bessel operator," Journal of Applied Mathematics and Stochastic Analysis, vol. 15, no. 3, pp. 277-286, 2002.

[17] S. Mesloub and N. Lekrine, "On a nonlocal hyperbolic mixed problem," Acta Scientiarum Mathematicarum, vol. 70, pp. 65-75, 2004.

[18] N. Mezouar and S. Boulaaras, "Global existence and decay of solutions of a singular nonlocal viscoelastic system with damping terms," Topological Methods in Nonlinear Analysis, vol. 11, 2020.

[19] S. Mesloub and S. A. Messaoudi, "Global existence, decay, and blow up of solutions of a singular nonlocal viscoelastic problem," Acta Applicandae Mathematicae, vol. 110, no. 2, pp. 705-724, 2010.

[20] D. Ouchenane, K. Zennir, and M. Bayoud, "Global nonexistence of solutions for a system of nonlinear viscoelastic wave equations with degenerate damping and source terms," Ukrainian Mathematical Journal, vol. 65, no. 5, pp. 723-739, 2013.

[21] M. A. Ragusa and A. Tachikawa, "Regularity for minimizers for functionals of double phase with variable exponents," Advances in Nonlinear Analysis, vol. 9, pp. 710-728, 2019.

[22] P. Shi and M. Shillor, "On design of contact patterns in one dimensional thermoelasticity," in Theoretical Aspects of Industrial Design, Philadelphia, PA, 1992.

[23] H. T. Song and D. S. Xue, "Blow up in a nonlinear viscoelastic wave equation with strong damping," Nonlinear Analysis, vol. 109, pp. 245-251, 2014. 
[24] H. T. Song and C. K. Zhong, "Blow-up of solutions of a nonlinear viscoelastic wave equation," Nonlinear Analysis: Real World Applications, vol. 11, no. 5, pp. 3877-3883, 2010.

[25] A. Zarai, A. Draifia, and S. Boulaaras, "Blow-up of solutions for a system of nonlocal singular viscoelastic equations," Applicable Analysis, vol. 97, no. 13, pp. 2231-2245, 2018. 